\title{
Corrigendum: Peroxisome proliferation-associated control of reactive oxygen species sets melanocortin tone and feeding in diet-induced obesity
}

Sabrina Diano, Zhong-Wu Liu, Jin Kwon Jeong, Marcelo O Dietrich, Hai-Bin Ruan, Esther Kim, Shigetomo Suyama, Kaitlin Kelly, Erika Gyengesi, Jack L Arbiser, Denise D Belsham, David A Sarruf, Michael W Schwartz, Anton M Bennett, Marya Shanabrough, Charles V Mobbs, Xiaoyong Yang, Xiao-Bing Gao \& Tamas L Horvath

Nat. Med. 17, 1121-1127 (2011); published online 28 August 2011; corrected after print 16 September 2011

In the version of this article initially published, the top electrophysiological trace of Figure 4a was inadvertently repeated as the bottom electrophysiological trace of Figure 4b. The scientific conclusions of the paper were not affected by the error. The error has been corrected in the HTML and PDF versions of the article.

\section{Corrigendum: Pharmacologic inactivation of kinase suppressor of ras-1 abrogates Ras-mediated pancreatic cancer}

H Rosie Xing, Carlos Cordon-Cardo, Xinzhu Deng, William Tong, Luis Campodonico, Zvi Fuks \& Richard Kolesnick Nat. Med. 9, 1267-1268 (2003); published online 07 September 2003; corrected after print 11 October 2011

In the version of this article initially published, there are irregularities with the tubulin loading controls in lanes 1 through 4 and with the KSR 1 bands in lanes 7 and 8 of Figure $2 f$. The authors have repeated the experiment and have provided a new figure panel that is now published as part of the correction notices linked to the HTML version and attached to the PDF version of the article. The original figures remain in both online versions of the article. The authors have also made a correction to Supplementary Figure 6b, which has been added to the supplementary file online. $\mathrm{H}$. Rosie Xing does not agree to this correction.

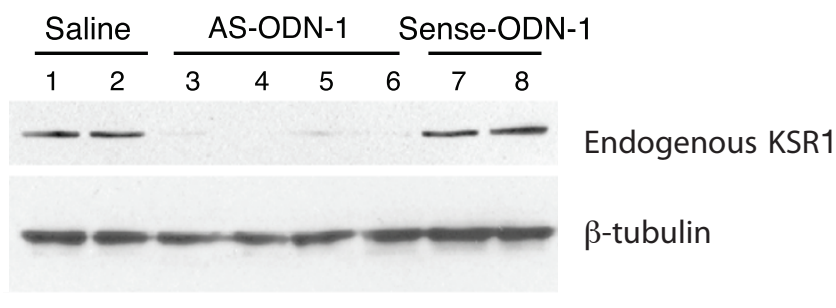

Figure $2 f$ 